\title{
Investigation on Visibility of Drilled Hole in Laser Micro-drilling of Multi-layered Artificial Skin
}

\author{
Yasuhiro Okamoto*, Kiichi Asako*, Naoto Maeda**, Akira Okada*, \\ Shogo Minagi**, Qiuyue Pan**, Keiji Jin**, Goro Nishigawa** \\ (Received Jan. 13, 2016)

\begin{abstract}
* Graduate School of Natural Science and Technology, Okayama University, 3-1-1 Tsushimanaka, Kita-ku, Okayama 700-8530, Japan

** Graduate School of Medicine, Dentistry and Pharmaceutical Sciences, Okayama University, 2-5-1 Shikata-cho, Kita-ku, Okayama 700-8525, Japan
\end{abstract}

\begin{abstract}
A multi-layered artificial skin is mainly consisted of three layers of silicone elastomer, print paper, and resin plate. Sealing of facial defects with the multi-layered artificial skin causes perspired water from the surface of missing parts, which results in insanitary conditions to the skin surface. Therefore, the laser micro-drilling using nanosecond pulsed laser of $266 \mathrm{~nm}$ was investigated to provide the breathability for multi-layered artificial skin without human eye's recognition of drilled holes, which can satisfy both the breathability and aesthetic problems. The visibility of drilled hole was affected by the pitch distance of drilled holes, and its visibility decreased with increasing the pitch distance. A small color difference measured using a colorimeter led to the low visibility, and laser micro-drilling with small color difference is effective to obtain the breathable multi-layered artificial skin with low visibility of drilled holes, which can improve the breathability for the multi-layered artificial skin.
\end{abstract}

Key words: micro-drilling, ns pulsed laser, multi-layered artificial skin, color difference

\section{INTRODUCTION}

Facial prosthesis treatment, which can repair missing parts of the face around caused tumors and traumatic injuries, has been attracted attention in the field of rehabilitation medical. Recently, a multilayered artificial skin, which is mainly consisted of three layers of silicone elastomer, print paper and resin plate, was newly developed ${ }^{1)}$. It was proposed by introducing the concept of personal digital shade guide and pseudo-angle layer as facial prosthesis in order to recover an aesthetic surface $\left.{ }^{2)}, 3\right)$. However, sealing of facial defects with the multi-layered artificial skin causes perspired water from the surface of missing parts, which results in insanitary conditions to the skin surface below the multilayered artificial skin. Therefore, air permeability is a desirable property of facial prosthesis, and microdrilling method with high breathability and good aesthetic quality is required for a multi-layered artificial skin, which can be applied to facial prosthesis treatment. In this study, fourth harmonics of Nd:YAG laser was used because of its high absorption rate to silicone and resin, and laser microdrilling of multi-layered artificial skin was experimentally investigated to provide the breathability for multi-layered artificial skin without human eye's recognition of drilled holes, which can satisfy both the breathability and aesthetic problems. Moreover, laser micro-drilled surface of multilayered artificial skin was aesthetically evaluated to characterize the appearance of laser drilled hole by a colorimeter and a color discrimination threshold experiment.

\section{SETUP OF LASER IRRADIATION EXPERIMENT}

Figure 1 shows a schematic diagram of the experimental setup. A Q-switched Nd:YAG laser pumped by a flash lamp was used as the laser source, and fourth harmonic of $266 \mathrm{~nm}$ of $5 \mathrm{~ns}$ pulse duration was used at constant pulse repetition rate of $20 \mathrm{~Hz}$ in this experiment. The intensity distribution and shape of the laser beam from the oscillator were unsuitable for the micro-processing experiment. Thus, in order to improve its intensity distribution and shape, the

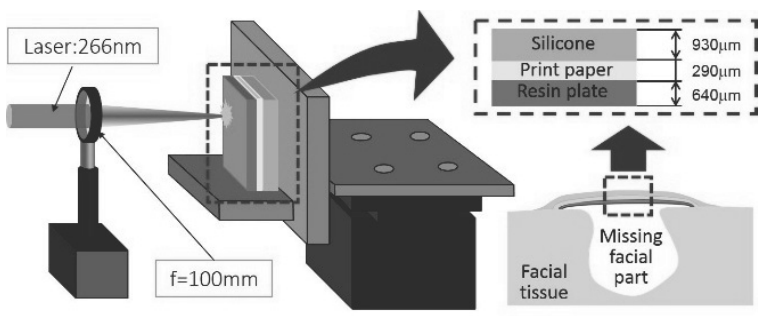

(a) Laser irradiation setup from (b) Multi-layered resin plate side irradiation artificial skin

Fig. 1 Schematic illustration of laser drilling experiment 


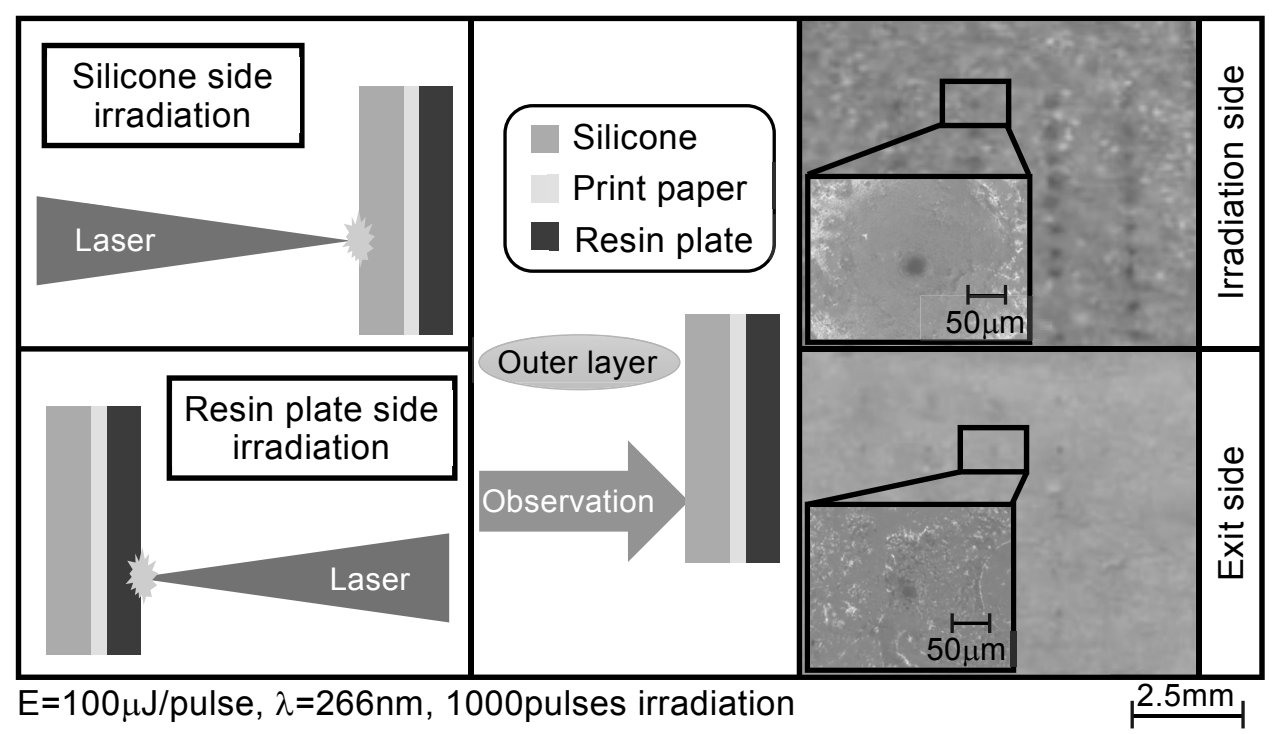

Fig. 2 Appearance of laser drilled holes on silicone elastomer side by $266 \mathrm{~nm}$

diameter of laser beam was increased by the upcollimator of 4 times, and this laser beam was formed using the circular mask of $0.1 \mathrm{~mm}$ in thickness. The diameter of the circular mask was calculated and selected in accordance with the wavelength of the laser beam to adjust the spot size of $50 \mu \mathrm{m}$ on the specimen surface using a focusing lens of $100 \mathrm{~mm}$ focal length as shown in Fig.1 (a). Gaussian mode laser beam, in which the intensity of laser beam is Gaussian distribution with the highest power at the center of beam, was obtained.

Figure 1 (b) shows a schematic illustration of multi-layered artificial skin, which is consisted of three structural layers. The tonal and textural appearances of the patient's skin are reproduced and matched with print paper by using the concept of individual shade guide ${ }^{1)}$. Multi-layered artificial skin is worn on the patient's facial surface by turns of resin plate (polycarbonate) as a scaffold material, print paper as a digital print layer, and silicone elastomer as an artificial cornified layer. Total thickness of specimen was $1.86 \mathrm{~mm}$, in which resin plate, print paper and silicone elastomer are $640 \mu \mathrm{m}$, $290 \mu \mathrm{m}$ and $930 \mu \mathrm{m}$ in thickness, respectively. Absorption rate of resin plate increases drastically less than $300 \mathrm{~nm}$ wavelength ${ }^{7)}$. In addition, the photon energy of laser beam increases with decreasing the wavelength, and it can be expected that laser beam of $266 \mathrm{~nm}$ wavelength can improve the processing performance compared with $1064 \mathrm{~nm}$ wavelength. Laser beam of $5 \mathrm{~ns}$ and $100 \mu \mathrm{J}$ was irradiated on multi-layered artificial skin in air.

\section{EFFECT OF LASER IRRADIATION DIRECTION ON AESTHETIC QUALITY}

Appearance of laser drilled hole on silicone elastomer affects aesthetic quality, which was investigated by changing the laser irradiation direction. Figure 2 shows photographs of laser drilled holes on silicone elastomer side, when 1000 shots of $266 \mathrm{~nm}$ were irradiated from silicone elastomer side and resin plate side. In these photographs, silicon elastomer side is irradiation side for silicone elastomer side irradiation, while resin plate side irradiation shows the exit side of drilled hole on silicon elastomer. In the case of silicone elastomer side irradiation, a wide heat affected area was observed as black circles around laser drilled holes. In contrast, their black areas were unclearly observed on silicon elastomer surface of multi-layered artificial skin in the case of resin plate side irradiation.

When the laser beam was irradiated from silicone elastomer side, the generation of laser induced plasma continued on silicone elastomer surface during laser irradiation time. Thermal effect of laser induced plasma attacked on the resin plate surface and inside of multi-layered artificial skin. Thermal decomposition temperature of silicone elastomer is around $820 \mathrm{~K}^{5), 6)}$, and it is well known that discoloration will occur around the drilled hole due to oxidation reaction by keeping higher temperature than the thermal decomposition one for a certain time. Its long heat input of the laser induced plasma into silicone elastomer and printed paper around the drilled hole occurred the heat accumulation, which resulted in the generation of a large heat affected zone. In contrast, proper laser irradiation time from resin plate side could minimize the time of heat input caused by the laser induced plasma, and heat affected zone around the drilled hole could be reduced. Therefore, the laser irradiation from resin plate side is effective in achieving good aesthetic 


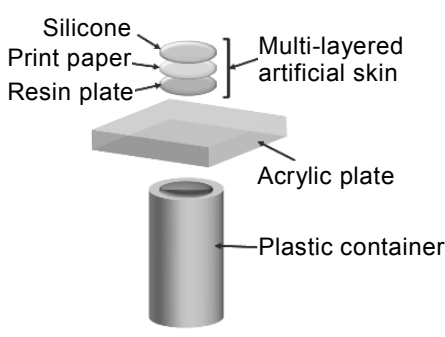

(a) Setup of specimen

(b) Setup of analyzer

Fig. 3 Setup of specimen and color analyzer for measuring color difference

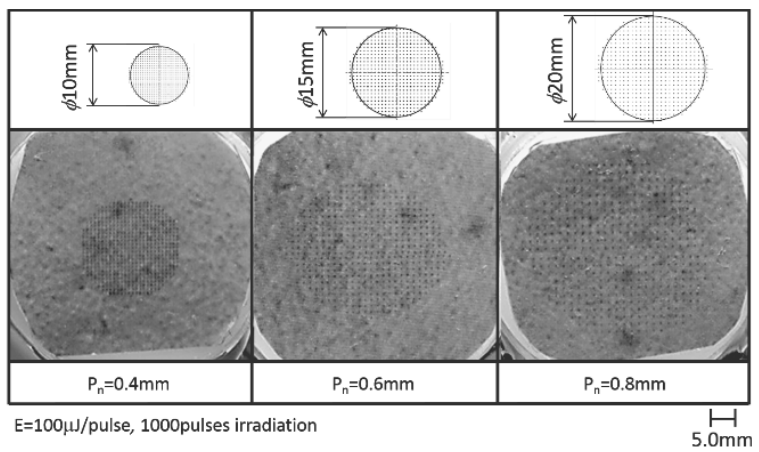

Fig. 4 Photographs of drilled holes on silicone elastomer surface as exit side by various hole pitches

quality of the laser drilled hole, and the irradiation direction was fixed from resin plate side in the following experiments.

\section{EFFECT OF PITCH DISTANCE OF DRILLED HOLES ON VISIBILITY AND COLOR DIFFERENCE}

Specimens were prepared as shown in Fig. 3 (a), and laser irradiation experiments from resin plate side were carried out by various pitch distances of drilled holes in order to discuss the visibility of laser drilled hole. Since the visibility of drilled hole would be influenced by the difference of color tone between hole parts and others, color difference of laser drilled specimen was evaluated by a colorimeter (TES corporation, TES-135 plus), which was put on the specimen as shown in Fig. 3 (b).

Figure 4 shows photographs of the drilled holes on silicone elastomer side, when laser beam of 266 $\mathrm{nm}$ and $100 \mu \mathrm{J}$ was irradiated from resin plate side by 1000 shots. Pitch distance of drilled holes $P_{n}$ was varied, and total number of drilled hole was constant to 532 at each pitch distance of drilled holes. Diameters of processed areas by hole pitch $P_{n}=0.4$ $\mathrm{mm}, 0.6 \mathrm{~mm}$ and $0.8 \mathrm{~mm}$ were $10 \mathrm{~mm}, 15 \mathrm{~mm}$ and

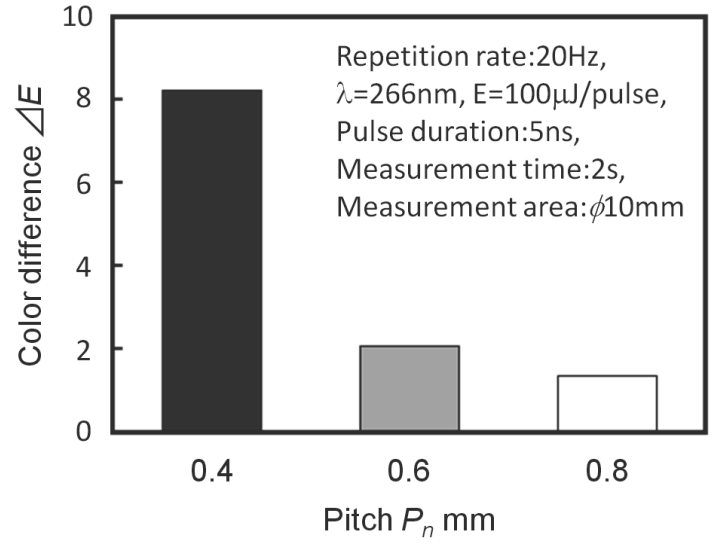

Fig. 5 Color difference of processed specimen with various pitch distances and non-processed specimen

$20 \mathrm{~mm}$, respectively. Laser drilled holes were arranged in a rectangular lattice shape with each hole pitch. As shown in the figure, marks of drilled hole become remarkable with decreasing the pitch distance of drilled holes, and they are inconspicuous at a large pitch distance of drilled holes.

In order to clarify the effect of pitch distance of drilled hole on the visibility of laser drilled hole, color discrimination was investigated at each hole pitch by using color difference $\Delta E$, which was measured as shown in Fig. 5. In general, color discrimination is defined as the recognition of color difference between plural color chromaticity and tone ${ }^{7)}$. In laser drilling process, discolored regions around drilled holes lead to the reduction of the brightness and the saturation, and it is considered that processing conditions change the color difference of processed specimen. Therefore, the color difference $\triangle E$ before and after drilling process was calculated by using brightness difference $\Delta L^{*}$ and chromaticity difference $\Delta a^{*}$, $\triangle b^{*}$ in Equation (1) ${ }^{7)}$.

$$
\Delta E=\sqrt{(\Delta L *)^{2}+(\Delta a *)^{2}+\left(\Delta b^{*}\right)^{2}}
$$

The measuring time and diameter of measured area are $2 \mathrm{~s}$ and $10 \mathrm{~mm}$, respectively. Since measurement area of $10 \mathrm{~mm}$ in diameter was equal to the diameter of minimum processed area, all processed specimen could be evaluated under the same condition. The average of 5 measurement values was recorded as the color difference $\triangle E$. As shown in Fig. 5, the color difference decreased with increasing the hole pitch, in other words, a larger hole pitch leads to similar color condition to non-processed specimen.

In order to evaluate the visibility of drilled hole, color discrimination threshold test was carried out as shown in Fig. 6. Observers watched the specimens 


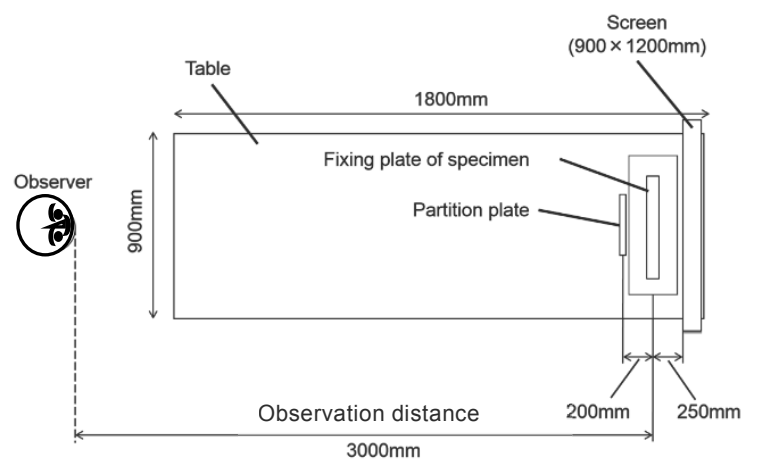

Fig. 6 Schematic diagram of color discrimination threshold test for processed specimen at the farthest distance of $3.0 \mathrm{~m}$

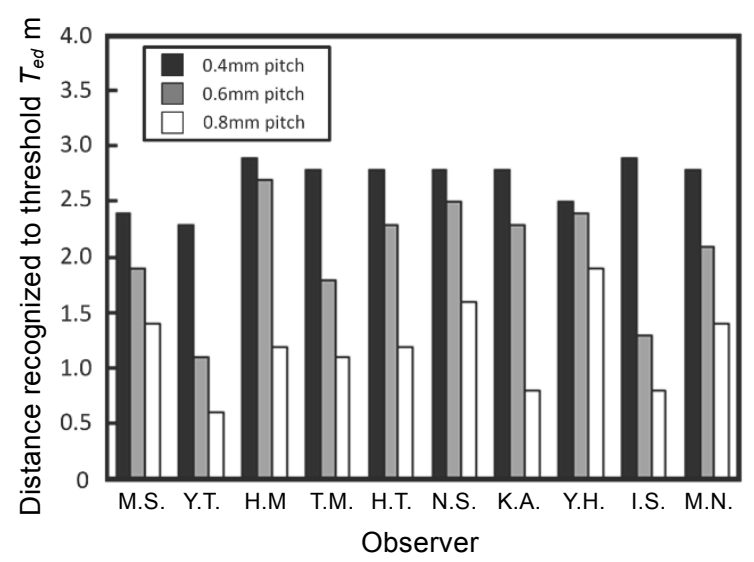

Fig. 7 Evaluation results of color discrimination threshold test for processed specimen with various pitch distances between drilled holes

with drilled holes for $5 \mathrm{~s}$ after removing the partition plate, and this observation was repeated at every 0.1 $\mathrm{m}$ observation distance from $3.0 \mathrm{~m}$. When observers recognized the drilled hole at a certain observation distance, its distance was recorded as the color discrimination threshold.

Figure 7 shows the evaluation results of color discrimination threshold test for processed specimen with various pitch distances of drilled holes by ten observers described as initials. Although there are individual differences in evaluation results, all observers indicated that color discrimination threshold decreased with increasing the pitch distance between drilled holes. These results agree well with measurement results of color difference as shown in Fig. 5. Since a large color difference between a specimen with and without drilled hole resulted in high visibility of drilled hole, laser microdrilling method with a small color difference is effective in reducing the visibility and improving aesthetic quality of drilled hole. Although skin color and condition vary individually, evaluation of color

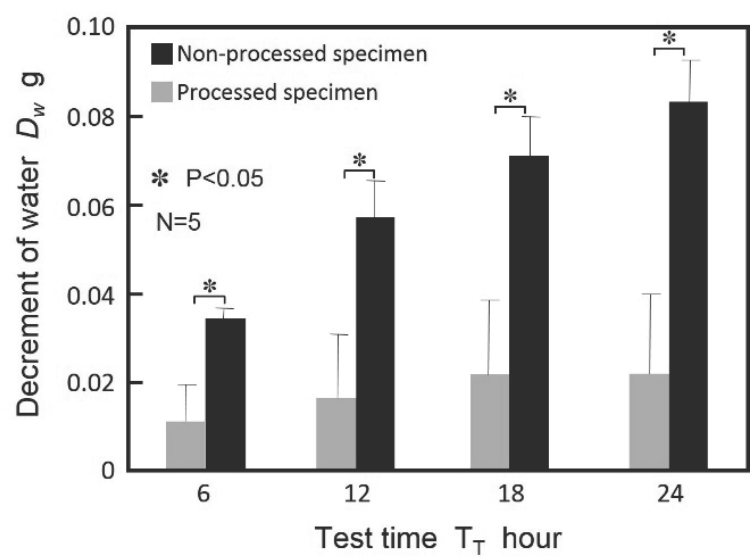

Fig. 8 Decrement of water with non-processed and processed specimen for various test time from 6 hours to 24 hours

difference is useful to discuss the visibility of laser drilled hole.

In normal, human would keep a certain distance from others to feel comfortable situation due to psychological effects ${ }^{8)}$, and it is reported that good interpersonal distances of comfortability and conversation are more than $1.5 \mathrm{~m}$ and $1.0 \mathrm{~m}$, respectively ${ }^{9}$. Therefore, it can be expected that multi-layered artificial skin with laser drilled holes can be applied to facial prosthesis treatment, although the laser drilled holes could not overcome human eye's recognition for the distance from others less than $1.5 \mathrm{~m}$.

\section{BREATHABILITY OF MULTI-LAYERED ARTIFICIAL SKIN WITH LASER DRILLED HOLES}

In order to discuss the breathability of multilayered artificial skin with laser drilled holes, test specimens were prepared, when the pitch distance of drilled holes and the diameter of processed area were $0.4 \mathrm{~mm}$ and $10 \mathrm{~mm}$, respectively. Distilled water was put in bottles, which were covered by multi-layered artificial skins with and without drilled holes. Weights of these test specimens were measured every 6 hours up to 24 hours in an incubator at 37 degree Celsius. Average weight difference of 5 test specimens was recorded as the decrement of water as shown in Fig. 8. Measurement results were statistically evaluated by using Mann-Whitney U test. A level of significant difference was considered as $5 \%(\mathrm{P}<0.05)^{10), 11)}$. Decrement of water of test specimen with drilled holes was larger than that without holes, and its decrement of water increased with increasing the test time. Therefore, it was clarified that fabrication of laser micro-drilled hole for the multi-layered artificial skin could improve the breathability. 


\section{CONCLUSIONS}

In this study, the laser micro-drilling using nanosecond pulsed laser was experimentally investigated to provide the breathability for multilayered artificial skin, and the visibility of drilled hole was discussed. Main conclusions obtained in this study are as follows:

(1) Heat affected zone around drilled hole caused by the laser irradiation on multi-layered artificial skin could be reduced by the laser irradiation from the resin plate side compared with that from the silicone elastomer side.

(2) Fabrication of laser micro-drilled hole for the multi-layered artificial skin could improve the breathability.

(3) The visibility of drilled hole on the multilayered artificial skin was affected by the pitch distance of holes, and its visibility decreased with increasing the pitch distance between laser drilled holes.

(4) The pitch distance of drilled holes had an influence on the color difference measured using a colorimeter, when the multi-layered artificial skin with drilled holes was compared with that without a drilled hole.

(5) Color discrimination threshold experiments revealed that the visibility decreased with increasing the pitch distance between laser micro-drilled holes.

(6) A small color difference measured using a colorimeter led to the low visibility, and laser micro-drilling with small color difference is effective to obtain the breathable multi-layered artificial skin with low visibility.

\section{REFERENCES}

1) Q. Pan, N. Maeda, K. Jin, S. Sakamoto, N. Kodama, G. Nishigawa, S. Minagi: Fabrication of Multi-layered Artificial Skin with the Concept of Artificial Cornified Layer and Individual Digital Shade Guide -For Quality Improvement and Simplification of the Fabrication of Facial Prosthesis-, The 30th Annual meeting of Japanese Academy of
Maxillofacial Prosthetics. Koriyama, Japan, (2013) p.43.

2) A. G. Wee, M. W. Beatty, D. J. Gozalo-Diaz, S. Kim-Pusateri, D. B. Marx: Proposed Shade Guide for Human Facial Skin and Lip: A Pilot Study. The Journal of Prosthetic Dentistry, No.110, No.2, (2013) p.82.

3) T. Nomura, J. Sato, M. Matsuura, K. Kawaguchi, R. Sekiguchi, A. Horie, K. Seto: Lightweight Acrylic Resin Facial Prosthesis for Maxillofacial Defects: A Fabrication and Retention Method. The Journal of Prosthetic Dentistry, Vol.110, No.4, (2013) pp.326-330.

4) S. Homma: Handbook about polycarbonate resin, Nikkan Kogyo Newspaper Publisshing, (1992) pp.366-367.

5) D. Bar-Long, C. Wen-Yen, L. King-Fu, S. F. Ming-Ren: Thermal Degradation Behavior of Epoxy Resin Blended with Propyl Ester Phosphazene, Journal of Applied Polymer Science, Vol.81, No.5, (2001) pp.1161-1174.

6) T. Ahamad, S. M. Alshehri: Thermal Degradation and Evolved Gas Analysis: A Polymeric Blend of Urea Formaldehyde (UF) and Epoxy (DGEBA) Resin, Arabian Journal of Chemistry, Vol.7, No.6, (2014) pp.1140-1147.

7) K. Uchida: Mechanism of Color Vision Contrivance to Recognize Color - Asakura Publishing, Tokyo, (1998) p.75.

8) S. Shibuya, A Study of the Shape of Personal Space, Bulletin of Yamanashi Medical University, No.2, (1985) pp.41-49.

9) K. Hashimoto, K. Nishida, K. Takahashi, T. Takahashi: A Study on the Psychological Territory Based on Experiments of Interpersonal Horizontal Distance, Journal of Architecture, Planning and Environmental Engineering No.485, (1996) pp.135-142.

10) Y. K. Cheung, J. H. Klotz: The Mann Whitney Wilcoxon Distribution using Linked Lists, Statistica Sinica, Vol.7, No.3, (1997) pp.805813.

11) E. Kasuya: Mann-Whitney U Test When Variances Are Enequal, Animal Behaviour, Vol.61, No.6, (2001), pp.1247-1249. 